\title{
THEORY OF SHOCK WAVES AND PLASMA WAVE-EMISSION
}

\author{
NICHOLAS A. KRALL \\ Laboratory for Applied Plasma Studies \\ Science Applications, Inc., La Jolla, Calif. 92037, U.S.A.
}

\begin{abstract}
The theory of shock waves in a hot plasma is reviewed, and the types of shock waves which can exist are summarized. The detailed application of shock wave theory to the explanation of laboratory experiments is described; a comparison is made between the results of these experiments and the observations made of type II radio bursts from the Sun.
\end{abstract}

\section{Introduction}

The papers presented at this meeting have made it clear that there is ample evidence for the existence of shock waves in flare-produced plasma, and suggests that those are responsible for a portion of the phenomena observed in type II solar burst activity.

The model usually cited (Wild and Smerd, 1972) is the following: a magnetic wave, moving across the coronal magnetic field, reaches a region where its speed exceeds the speed of magnetic sound. At that point it steepens, and forms a magnetic shock wave. This wave produces a density compression and an electron current, and, most important, is unstable to the production of electron plasma oscillations. These plasma oscillations (frequency $\omega_{p}$ ) scatter off ion density fluctuations to produce the observed electromagnetic radiation at frequency $\omega_{p}$, and scatter off each other to produce the radiations observed at frequency $2 \omega_{p}$. Evidence for this model includes the fact that sources of type II bursts move at Alfven speeds, and that the emission is too strong to be thermal.

In this review I will attempt to do three things. First, review the theory of how (and what kind of ) shock waves can exist in a hot plasma, second show what sorts of detailed calculations are now possible in shock wave theory (and what phenomena they have uncovered) and third describe controlled laboratory experiments which coincide well enough with the theory to convince us that we are still on the right track; in large amplitude wave theory the effects are so strongly nonlinear that only such comparison allows us to proceed with much hope that we haven't forgotten some basic effects or made some hopelessly bad approximations. In the course of this talk I will try to convince you that laboratory experiments are seeing many of the same effects that solar physicists see, and that our ability to calculate these effects in detail for laboratory applications gives promise of similar success in coronal shock theory.

\section{Classical Theory of Shock Waves in Collisionless Plasma}

There is a well established theory of macroscopically stable shock waves in plasma (Tidman and Krall, 1971a), with mechanisms and classification schemes which are 
widely accepted. In this section I will briefly review these ideas, to set up a framework in which more recent theoretical and experimental results can be viewed.

We use the word shock wave in a very loose sense, meaning simply a situation in which the plasma changes state during the passage of a transition region. The change of state may be smooth (laminar) or turbulent. The change of state across the shock front in most cases of solar interest does not take place as a result of collisions, but rather because of collective effects. That is, plasma instabilities take energy from the ordered streams and fields of the shock and dump it into more nearly random energy. I resist saying that the energy goes into temperature. In fact, the energy often goes into motion on a shorter length scale and higher frequency scale than that of the shock itself. On the shock scale this may in fact look like temperature, but on a microscopic scale there has been no increase in entropy; rather, a large number of high frequency plasma waves have been generated, with the particles sloshing about in the troughs of these waves. Thus the conservation laws (Tidman and Krall, 1971b), (Rankine-Hugoniot relations) which connect the unshocked plasma state with the shock state, must include the electrical energy of the plasma waves in the shocked plasma, and must take account of the fact that the ions and electrons respond differently to the waves.

The greatest progress in classifying and studying shock waves has been to study under what conditions laminar solutions are possible (Sagdeev, 1966). In these cases, the plasma properties can be divided into two camps. First are the slowly and smoothly varying (i.e. laminar) quantities, such as the density $N(x, t)$, the magnetic field $B(x, t)$, the electric field $E(x, t)$ etc. Second are the more rapidly fluctuating quantities $\delta N(x, t), \delta B, \delta E$, which are treated as perturbations on the laminar parameters. Thus,

$$
N_{\text {Total }}=N(x, t)+\delta N(x, t)
$$

and so on.

The laminar quantities are governed by the fluid equations of continuity and momentum transfer and by Maxwell's equations. It is generally necessary to treat the electrons and ions as separate fluids.

Laminar shock waves come out of the theory as follows: One possible motion of an electron-ion fluid is a low amplitude wave. Of the various wave motions of a plasma, many (for example: magnetosonic waves, ion acoustic waves, whistler waves) are sound-like over some frequency range, as shown in Figure 1. If the wave is of finite amplitude, nonlinear terms $(\mathrm{V} \cdot \nabla \mathrm{V})$ in the fluid equations will couple a wave $(\omega, k)$ to a higher harmonic $(2 \omega, 2 k)$. In the soundlike portions of the wave spectrum the harmonics travel at the same speed as the original wave. Since they are of shorter wavelength, they represent wave steepening. A shock forms when the steepening is balanced by some other mechanism, leading to a transition with a fixed transition width. One way to balance nonlinear steepening is by dispersion. When a harmonic is generated at a sufficiently short wavelength (high $k$ ) it no longer propagates with the same speed as the rest of the pulse; this phenomenon is called dispersion. It comes about 
because in general soundlike waves $\omega=k V_{s}$ only occur in some appropriate limit such as a plasma with zero mass electrons, or a plasma in which $N_{e}=N_{i}$ everywhere. Short wavelength or high frequency harmonics sense the actual electron mass, or the actual charge separation, and fall behind the longer wavelength lower frequency modes (in some cases they speed ahead of the main pulse, for a wave whose dispersion curve is of the type b in Figure 1). Dispersion will not give a shock wave by itself, but
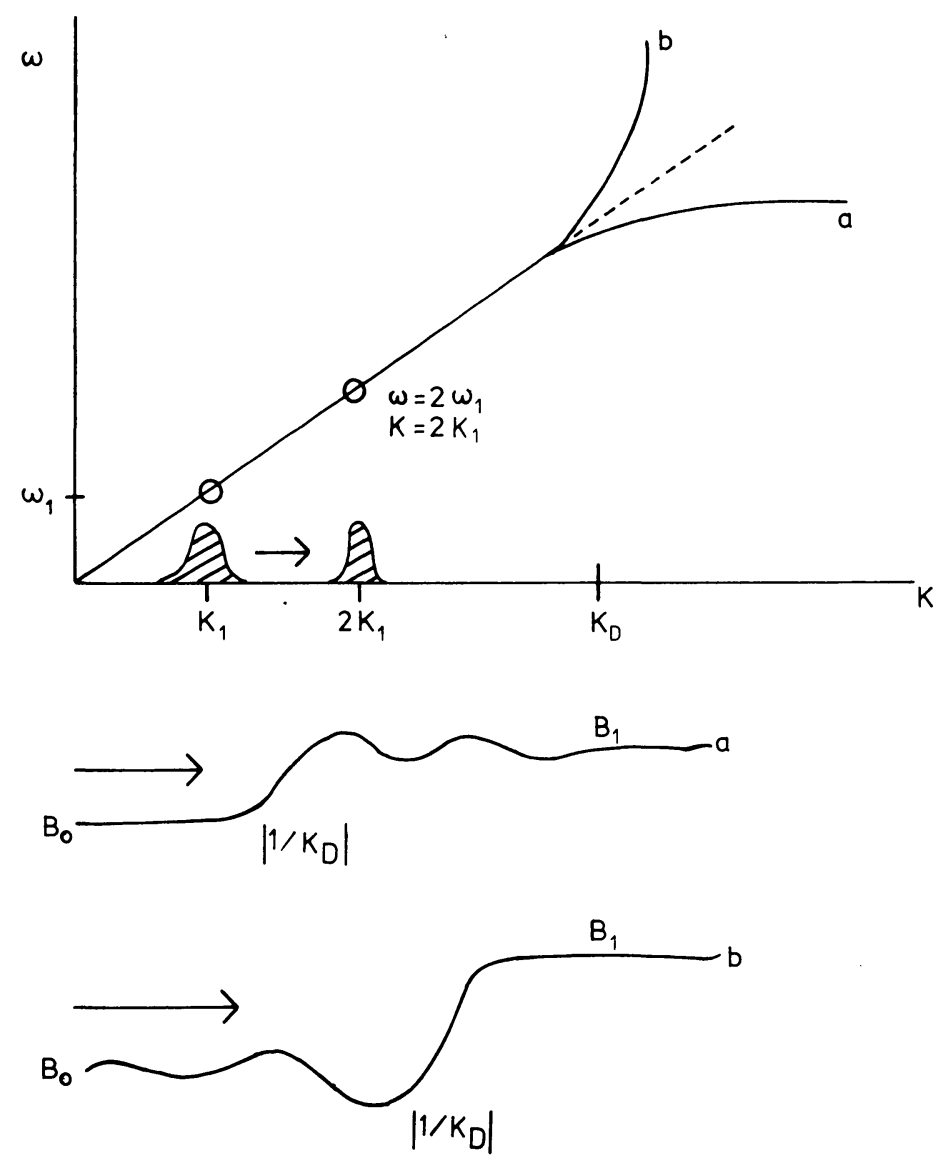

Fig. 1. Typical dispersion curves for sound-like waves in plasma.

will limit the steepening and form a large amplitude fixed profile wave, often called a soliton, or solitary wave. To convert this into a shock requires a dissipation mechanism, such as Landau damping or a plasma instability driven by currents in the soliton. Such a shock wave will have a thickness $L_{s} \simeq k_{\mathrm{D}}^{-1}$ (see Figure 1). If the instabilities are strong enough, they can themselves provide enough dissipation to limit the steepening, by acting as a drain on the currents and fields which would build up in a very steep shock. In this case the dispersive width would never be reached, $L>1 / k_{\mathrm{D}}$. Experimentally, this has proved to be the rule rather than the exception. 
A third possibility is that the steepening is so rapid that it cannot be limited by either dispersion or dissipation. In this case the wave 'breaks', in much the same way that a water wave breaks (in fact, the whole shock picture described above has an exact mathematical analogy to a water wave approaching a beach). The subsequent fate of the wave cannot be calculated, and depends on the coupling of the spilling wave to the background medium. Because the steepening rate increases with wave amplitude and Mach number $\mathscr{M}=$ (shock speed)/(linear sound wave speed), the breaking point defines a critical Mach number $\mathscr{M}_{c}$ for each type of shock wave. In practice, $\mathscr{M}_{c}$ can only be calculated when dissipation is neglected, or when some very specific and simplified model is adopted to describe the dissipation.

With that preamble I will describe laminar shock waves most likely to be involved in burst phenomena, and their stability, and give a general diagram of possible laminar shocks.

\subsection{Magnetosonic SHOCK Waves}

These waves propagate across a magnetic field, $B=B_{z}(x)+B_{z 0}$, and have a linear dispersion relation given by

$$
\omega=\frac{k V_{\mathrm{A}}}{\left(1+k^{2} c^{2} / \omega_{p}^{2}\right)^{1 / 2}},
$$

where $\omega$ and $k$ are the frequency and wave number, $V_{\mathrm{A}}=B /(4 \pi N M)^{1 / 2}$ is the Alfvén speed, $\omega_{p}=\left(4 \pi N e^{2} / m\right)^{1 / 2}$ is the plasma frequency, $c$ is the speed of light, $N$ the plasma density and $(m, M)$ the (electron, ions) mass. Thus their dispersive thickness is

$$
L_{s} \simeq\left(c / \omega_{p}\right) /(2 \sqrt{\mathscr{M}-1}),
$$

their Mach number is (with $V$ the shock speed)

$$
\mathscr{M}=V / V_{\mathrm{A}} \text {. }
$$

It can be shown that the shock amplitude and compression are

$$
\begin{aligned}
\frac{B}{B_{0}} & =2(\mathscr{M}-1) \\
\frac{\Delta N}{N} & =\frac{\Delta B}{B_{0}}\left[1-\frac{\Delta B}{2 B}\right]^{-1} .
\end{aligned}
$$

The shock breaks at $\Delta B=2 B$. Note that $\Delta N / N \rightarrow \infty$ at that point ( $B$ is so strong that all the plasma is about to be reflected forward, ahead of the shock). Adding a resistivity $v$ to the fluid equation may allow a higher amplitude shock, depending on the model used for $v$. Constant resistivity, for example, doesn't help.

Because the front of this shock contains a current carried primarily by electrons,

$$
\mathbf{J}=-N e \mathbf{V}_{e}=\frac{c}{4 \pi} \nabla \times \mathbf{B}
$$


it can be unstable to a variety of plasma oscillations if it is steep enough. In particular, the conditions to drive a strong two-stream instability is that

$$
\mathscr{M}>1+\left(\frac{8 \pi N T_{e}}{B^{2}}\right)^{1 / 3} \text {. }
$$

This shows that low $\beta\left(=8 \pi N T / B^{2}\right)$ shocks are easily unstable to the emission of plasma waves (Krall and Trivelpiece, 1973).

\subsection{ShOCK WAVES PARALlel to $B_{0}$}

Because existing models of shock wave produced type II bursts often assume shock waves propagating across $B_{0}$, as described above, I choose as a second wave example the whistler soliton which propagates parallel to the ambient magnetic field. This mode could, for example, propagate parallel to a neutral sheet, or along a magnetic streamer.

This wave propagates in the direction of $B_{0}$, has an electric field vector in the same direction, while the shock magnetic fields $B_{\perp}$ are perpendicular to $B_{0}$. The characteristic width of the shock is determined by electron-inertia produced dispersion,

$$
L_{s} \approx \frac{c B_{0}}{\omega_{p i} B_{\perp}}
$$

where $\omega_{p i}$ is the ion plasma frequency. The range of amplitudes and speeds over which this soliton propagates is

$$
\begin{aligned}
0 & <B_{\perp}<B_{0}(M / m)^{1 / 2} \\
V_{\mathrm{A}}(M / 4 m)^{1 / 2} & <V<V_{\mathrm{A}}(M / 2 m)^{1 / 2} .
\end{aligned}
$$

A further restriction on the amplitude of the shock comes from a quasi-neutrality assumption used to derive it, requiring that

$$
B_{\perp}<B_{0}(M / m)^{1 / 2}\left(\frac{m}{M} \frac{\pi N m c^{2}}{B_{0}^{2} /{ }^{8} \pi}\right)^{1 / 4}
$$

The shock electric field $E$ induces electron currents in the shock front, which can drive electron plasma waves unstable, just as for the magnetosonic shock wave. The shock strength needed to drive this instability is

$$
\frac{B_{\perp}}{B_{0}}>\left(\frac{4 \pi N T_{e}}{B_{0}^{2}}\right)^{1 / 4}\left(\frac{M}{m}\right)^{1 / 4}
$$

For a low $\beta$ region, or a region of relatively small $B_{0}$, this is a condition that can realistically be met, and gives a mechanism for motion of type II bursts along the magnetic lines. 
The two examples given here are merely indicative of the wide variety of possible laminar shock structures. Many more are summarized and tabulated in Wild and Smerd (1972), and I refer the audience to that monograph for further details.

\section{Laboratory Results Related to Flare-Produced Shock Waves}

As I mentioned several times already, type II burst models are usually based on shock waves traveling across a magnetic field. There is a class of laboratory experiments which uniquely produce large amplitude magnetic waves traveling across $B_{0}$, and it is useful to see to what extent they produce the same phenomena found in the corona, and to what extent they can be used to sort out various burst models. This experiment is called a $\theta$-pinch, because of its geometry (Hintz, 1970). A cylinder of plasma confined by a magnetic field $B_{z}$ is subjected to a pulsed field $\delta B_{z}$ at its boundary (this field is produced by a current carrying coil wrapped around the cylinder. The current is in the $\theta$ direction, hence the name of the experiment). The pulsed field creates a magnetic disturbance which propagates across the magnetic field, at speeds similar to the Alfvén speed. A magnetic shock often forms. The parameters of this experiment which make it relevant to the corona are

$$
\begin{aligned}
& \beta=\frac{N T}{B^{2} / 8 \pi}<1 \\
& 4 \pi N m c^{2}>B^{2} \\
& a_{i}>L_{s}>a_{e}>\lambda_{\mathrm{D}} \\
& V_{S} \simeq B_{0} /(4 \pi N M)^{1 / 2},
\end{aligned}
$$

where $\lambda_{\mathrm{D}}$ is the Debye length and $\left(a_{e}, a_{i}\right)$ are the (electron, ion) gyroradii. This is the same ordering of the relevant parameters found in the corona.

Figure 2 shows a plot of the magnetic profile at one instant of time in the experiment. The case shown had $\delta B$ antiparallel to $B_{0}$; both parallel and antiparallel cases have been thoroughly studied. The figure lists some of the plasma processes which we believe are going on during the magnetic field implosion. These are clearly the same sort of features mentioned in burst models.

Some of the results of the most recent $\theta$-pinch experiments (Davis et al., 1970; ChinFatt, private communication; Chin-Fatt and Griem, 1970) are the following:

(1) Emission of radiation of $\omega_{p}$ and $2 \omega_{p}$

(2) Proton acceleration to $V \sim 2 V_{\text {shock }}$

(3) Plasma Wave Generation

(4) Electron Heating to $10^{7}-10^{8}{ }^{\circ} \mathrm{C}$

(5) Ion Wave Generation

In particular, the characteristics of the $\omega_{p}, 2 \omega_{p}$ emission were (Chin-Fatt, private communication; Chin-Fatt and Griem, 1970).

(1) Emission was consistent with a bi-Maxwellian electron distribution, with the hot component 100 times as hot as the cold component 
(2) Emission peaked late in time, and presumably came from a region behind the shock

(3) The emission level was about $10^{3}$ above thermal

(4) The strengths of the two components were of the same order of magnitude

(5) The presence of ion waves was also inferred

Clearly these, particulatly the $\omega_{p}$ and $2 \omega_{p}$ emission, are some of the main features of

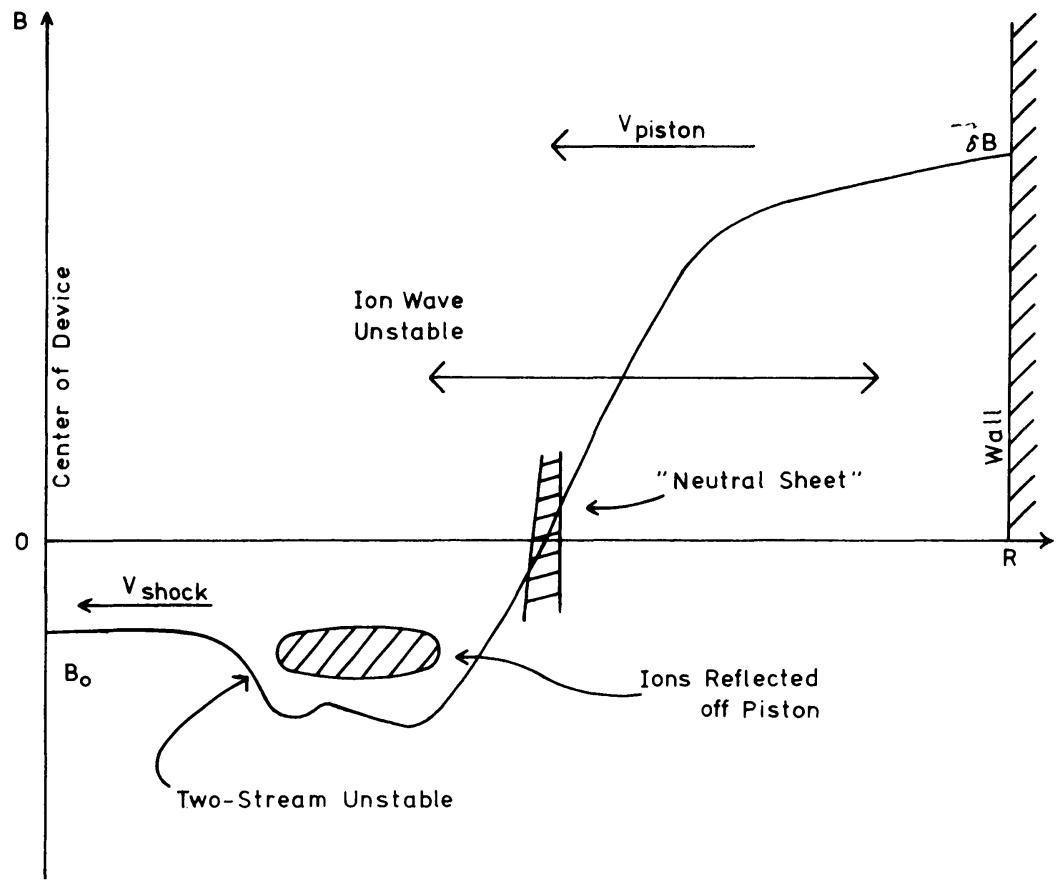

Fig. 2. Magnetic profile during magnetic implosion in a $\theta$-pinch experiment.

the radio burst observations, and include as well direct measurement of other properties which have so far been merely the subject of speculation.

For example, the long time duration and late peaking of both the fundamental and the harmonic radiation argues against models (Saitsev, 1969) in which a coherent set of solitary waves in adjoining plasma layers give forward and backward plasma waves which scatter to give $2 \omega_{p}$. A more plausible idea is that the plasma wave generated by instability in the shock front have isotropized by various diffusion processes in $k$-space (this includes the idea (Tidman and Krall, 1971a ; Papadopoulos, 1969) that the particle distribution behind the shock interacts with the turbulence to develop a non-Maxwellian number of energetic particles leading to a superthermal level of plasma wave emission). 


\section{Recent Theoretical Advances}

The ability to explain theoretically the results of $\theta$-pinch experiments cited above offers hope for similar success in explaining coronal phenomena. The things which are well understood are the laminar shock wave structure as discussed in Section 2, the conditions under which linear instabilities can be excited, the initial rates at which instabilities take energy from the electron current, and the equipartition of that energy among electrons, ions and plasma waves. These ideas and the details of their calculation are listed in a variety of plasma texts, e.g. Krall and Trivelpiece (1973), and in previous reviews of radio burst phenomena (Wild and Smerd, 1972; Smith, 1971). The most recent theoretical work on this problem has been to apply the known stability theory in a practical and selfconsistent way. It is not often fair to assume that a magnetic pulse will develop into a shock which has a time-stationary profile determined from laminar theory, and then use this profile to calculate instabilities and plasma wave emissions. Clearly the instabilities will act on the plasma reducing the current, heating the plasma, and altering the profile which caused the instability in the first place. One recent approach to this problem has been to use the two-fluid equations to describe a plasma in which a magnetic pulse is applied at a boundary. The two fluid equations include self consistently the effect of a variety of instabilities, which appear in the form of time and space dependent turbulencedetermined transport coefficients. These include, for example, the drag on the current due to plasma wave emission and the effective heating of electrons by the plasma waves. 'Self consistent' means that, for example, if the plasma conditions say that the plasma is unstable, the resistivity begins to grow at the rate given by linear stability theory; if the instability heats the plasma and quenches itself, the resistivity returns to its stable-plasma value. In this framework, the fluid equation for electron momentum transport is

$$
\begin{aligned}
N\left(\frac{\partial}{\partial t}+\mathbf{V} \cdot \nabla\right) \mathbf{V} & =\frac{-N e}{m}\left(\mathbf{E}+\frac{\mathbf{V} \times \mathbf{B}}{c}\right)-\frac{\nabla N T}{m}-\frac{e}{m}\langle\delta N \delta \mathbf{E}\rangle \\
\delta N & =N_{0} e^{i \omega t} e^{\gamma t} \\
\delta \mathbf{E} & =\mathbf{E}_{0} e^{i \omega t} e^{\gamma t} \\
\gamma & =\gamma(x, t, \mathbf{V}, T),
\end{aligned}
$$

where $\langle>$ means an average over the rapidly varying instability variables and the growth of the instability $\gamma$ is determined by the conditions actually present locally in the plasma. The reason this calculation is possible is that computer techniques now allow numerical integration of the nonlinear two fluid equations.

This approach has had marked success in predicting the rate of penetration of magnetic pulses into a plasma, the magnetic profile (which may or may not resemble laminar solutions), the plasma heating, and the rate and regions of production of plasma waves. Figure 3 shows our calculation of the penetration time of a magnetic pulse into a field free plasma, as a function of plasma density, and plasma species. The 
lines are experimental results (Bengston et al., 1972), the points are calculated by numerical integration of the turbulent-transport fluid equations. Similar success is achieved for scaling with the pulse field $B_{\max }$. Note that since this is penetration into a field free plasma, the Mach number would be infinite. Laminar theory is helpless in this situation. In fact, a shock does not develop in the lifetime of the experiment, but neither does the pulse steepen and break. Turbulent resistivity and viscosity allow

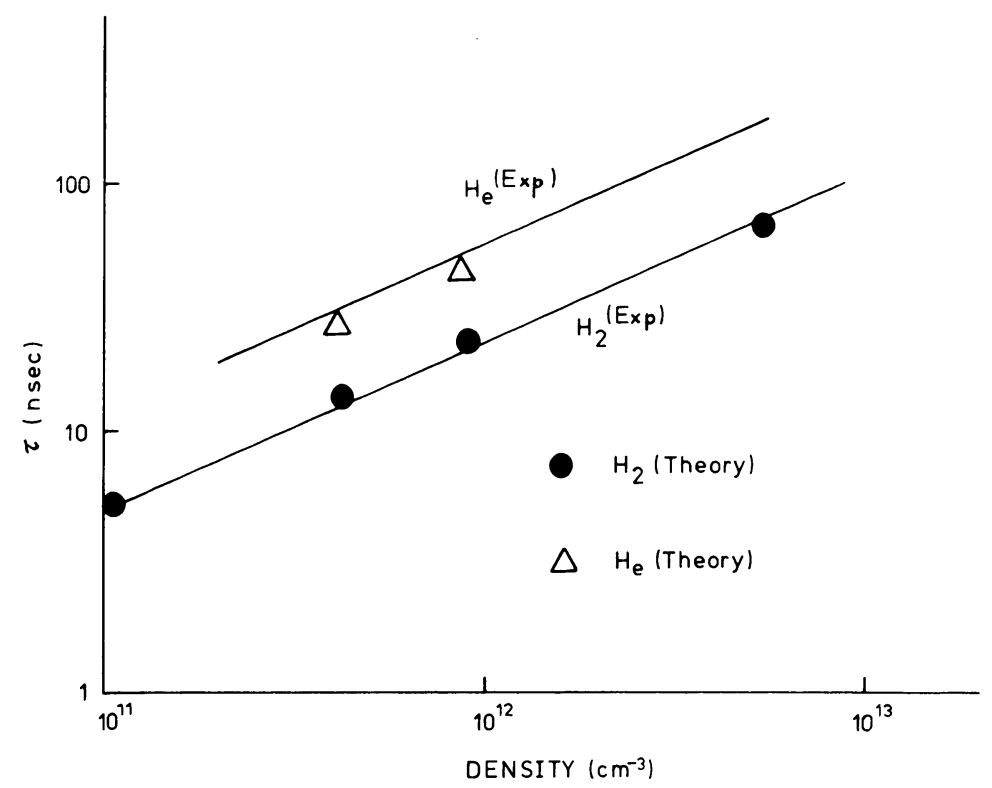

Fig. 3. Theory vs experiment for the penetration time $\tau$ of a magnetic pulse into a field free plasma.

the pulse to penetrate in a well organized fashion to the center of the machine. Figure 4 shows a typical magnetic profile during the run, and shows the spatial region in which plasma waves are excited. Note that this is a well defined region. Further into the pulse electron heating has quenched the instability. Radio emissions from these plasma waves would be more characteristic of the plasma density in the unshocked region than of the shocked plasma. This ability to perform calculations beyond the range of laminar theory and to extract real detail about heating and wave production is a hallmark of our model and offers hope that it can be extended to corona calculations.

A number of useful quantities have not been calculated, because they were not of laboratory interest, yet their calculation should be possible. For example, since the turbulent fields are known, the level of electromagnetic emission at $\omega_{p}$ could be calculated. Further, using the same fields, stochastic acceleration could also be calculated, either as a mechanism to accelerate particles out of the shock or as a mechanism to generate superthermal tails in the electron distribution $f_{e}$ required by some models to generate more plasma waves.

Thus, it seems that many effects studied in the laboratory are directly relevant to 


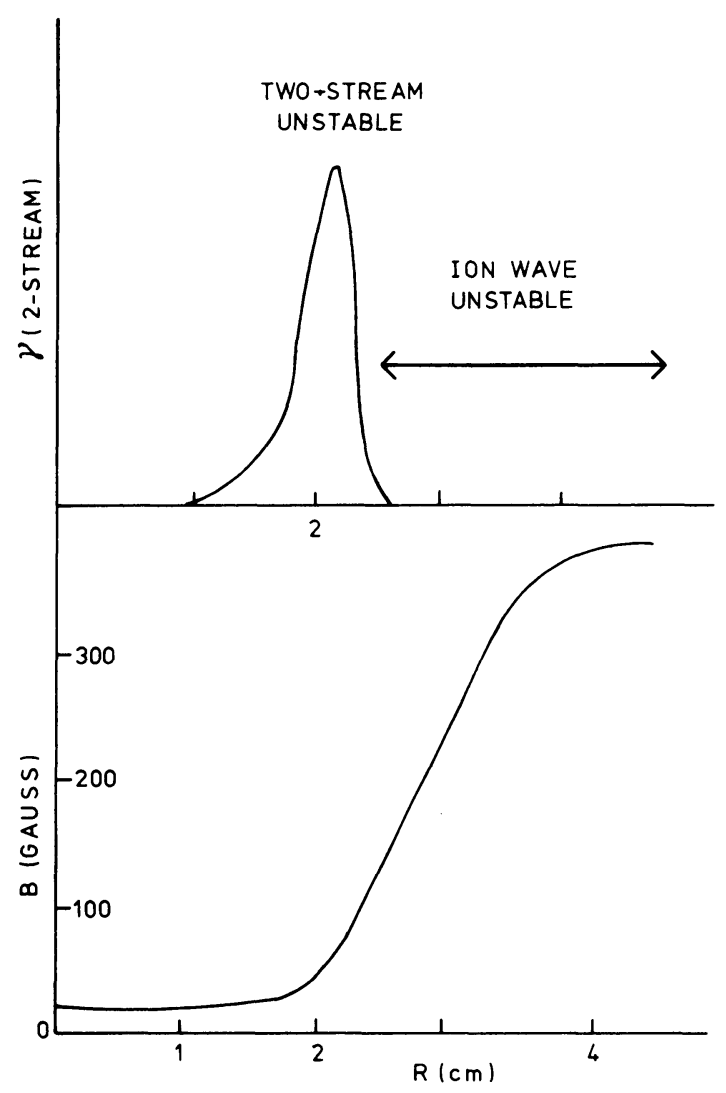

Fig. 4. Computer prediction of the two stream unstable region during penetration of a magnetic pulse.

explanation of solar corona phenomena, and collaboration between specialists of these two fields should be increasingly fruitful, particularly if the astrophysical data and laboratory theory continues to show such rapid improvement.

\section{Acknowledgements}

It is a pleasure to acknowledge an illuminating discussion with Dr D. A. Tidman on the facts and fictions of the solar burst problem. This work was performed while the author was a fellow of the John Simon Guggenheim Memorial Foundation.

\section{References}

Bengston, R. D., Marsh, S. J., Robson, A. F., and Kapetanakos, I. A.: 1972, Phys. Rev. Letters $29,1073$. Chin-Fatt, C. and Griem, H. R.: 1970, Phys. Rev. Letters 25, 1644.

Davis, N., DeSilva, A. W., Dove, W., Griem, H. R., Krall, N. A., and Liewer, P. C.: 1971, in Proc. 4th International Conference on Plasma Physics and Controlled Nuclear Fusion Research, IAEA Vienna.

Hintz, E.: 1970, in H. Griem and R. Lovberg (eds.), Methods of Experimental Physics 9A, 213. 
Krall, N. A. and Trivelpiece, A. W.: 1973, Principles of Plasma Physics, McGraw-Hill, New York.

Liewer, P. C. and Krall, N. A.: 1974, Phys. Fluids, in press.

Papadopoulis, K. : 1969, Phys. Fluids 12, 2185.

Sagdeev, R. Z.: 1966, in M. A. Leontovich (ed.), Reviews of Plasma Physics, Vol. 4, Consultants Bureau, New York.

Smith, D. F.: 1971, Astrophys. J. 170, 559.

Tidman, D. A.: 1965, Planetary Space Sci. 13, 781.

Tidman, D. A. and Krall, N. A.: 1971a, Shock Waves in Collisionless Plasmas, Wiley, New York, and references therein.

Tidman, D. A. and Krall, N. A.: 1971b, Shock Waves in Collisionless Plasmas, Wiley, New York, pp. 8-12. Wild, J. P. and Smerd, S. F.: 1972, Ann. Rev. Astron. Astrophys. 10, 159.

Zaitsev, V. V.: 1969, Soviet Astron. AJ 12, 610.

\section{DISCUSSION}

Dryer: It has been precisely for the reasons discussed by Dr Krall that two years ago we initiated a detailed study of interplanetary structures which appeared to be associated with either flares or stream interactions. We wanted to define the shocks in terms of the parameter discussed by Dr Krall as part of the overall study of the structure itself (piston and any other discontinuities). We have started at the lowest level of sophistication, namely use of the fluid Rankine-Hugoniot equations, together with Maxwell's equation (i.e., the De-Hoffman-Teller level). Using least square fitting to suspected shocks, we have computed all parameters (shock, normal direction and velocity, etc.). The two slides show the magnetic data for a reverse shock (normal pointed back toward the sun) which moved first past Pioneer 9 - at $0.13 \mathrm{AU}$ upstream of Earth and then past OGO-5 (at Earth). The slides show the preservation if its identity; the fits to the data show that it is a supercritical, highly oblique, high-beta shock. Its signature is (in the magnetic field) characteristic of a perpendicular shock as indicated by collisionless shock theory.

Sturrock: One of the puzzles regarding type II and III bursts is that II's radiate at both fundamental and harmonic while III's radiate predominately at the harmonic. Shocks produce ion-acoustic waves, and thus we should except type II's to be dominant in the fundamental.

Krall: Ion-acoustic waves are delicate and not always seen even in the lab. They are an important source of heating in the lab situations.

Smith (to Sturrock): This is a reasonable possibility because we don't generate ion-acoustic waves in type III bursts.

Smith (to Krall): The reason we worry about shocks going along field lines is that we don't know how to generate plasma radiation from such shocks. Do you have any idea how to produce such radiation from a parallel ion-acoustic shock?

Krall: There are differences in velocity between electrons and ions in such a shock and thus there may be such a possibility, but it has not been analyzed.

Smith: What is the minimum Alfvén Mach number $M_{\mathrm{A}}$ for production of plasma radiation in the Maryland experiment you described?

Krall: $M_{\mathrm{A}}=2-3$, but this may not necessarily be a lower limit because of experimental conditions. 